\title{
Increased expression of TNFRSF14 indicates good prognosis and inhibits bladder cancer proliferation by promoting apoptosis
}

\author{
YU-DI ZHU ${ }^{1}$ and MING-YUE LU ${ }^{2}$ \\ ${ }^{1}$ Department of Urology, Ningbo No. 2 Hospital, School of Medicine, Ningbo University, Ningbo, Zhejiang 315010; \\ ${ }^{2}$ Department of Oncology, First Affiliated Hospital of Jinan University, Guangzhou, Guangdong 510630, P.R. China
}

Received December 4, 2017; Accepted May 23, 2018

DOI: $10.3892 / \mathrm{mmr} .2018 .9306$

\begin{abstract}
Despite advances in management, bladder cancer remains a principal cause of cancer-associated complications. Tumor necrosis factor receptor superfamily member 14 (TNFRSF14) is dysregulated in certain types of cancer; however, limited data are available on the expression and function of TNFRSF14 in bladder cancer. In the present study, the aim was to evaluate the expression and biological functions of TNFRSF14 in bladder cancer. Firstly, the expression levels of TNFRSF14 in bladder cancer tissue were examined using The Cancer Genome Atlas (TCGA) database. Secondly, reverse transcription-quantitative polymerase chain reaction was utilized to investigate the expression levels of TNFRSF14 in the T24, SW780 and EJ-M3 bladder cancer cell lines. Transfection and Cell Counting kit-8 (CCK-8) assay was used to evaluate whether TNFRSF14 overexpression or silencing would have an effect on cell proliferation of T24 and EJ-M3 cells. In addition, TNFRSF14-induced apoptotic cells were identified using Annexin V-fluorescein isothiocyanate and propidium iodide staining. Western blot analysis was used to detect proteins associated with the phosphatidylinositol 3-kinase pathway. According to the TCGA dataset, the expression levels TNFRSF14 were decreased in bladder cancer tissue compared with in normal control samples. Patients with bladder cancer exhibiting low expression levels of TNFRSF14 had a worse prognosis compared to those with high expression levels of TNFRSF14. Overexpression of TNFRSF14 in T24 cells led to increased apoptosis and inhibited cell proliferation in vitro. Western blotting demonstrated that TNFRSF14 overexpression increased the expression levels of caspase3-p17 in T24 cells, but significantly decreased the expression levels of phosphorylated (p)-protein kinase B (AKT) and P70 S6 kinase (P70). TNFRSF14 silencing in EJ-M3 cells enhanced
\end{abstract}

Correspondence to: Dr Ming-Yue Lu, Department of Oncology, First Affiliated Hospital of Jinan University, 613 West Huangpu Avenue, Guangzhou, Guangdong 510630, P.R. China E-mail: m18927999685@163.com

Key words: tumor necrosis factor receptor superfamily member 14, bladder cancer, cell proliferation, apoptosis, prognosis cell growth, inhibited cell apoptosis, increased the expression levels of p-AKT and P70, and decreased the expression levels of caspase3-p17. In conclusion, TNFRSF14 may serve a tumor suppressive role in bladder cancer by inducing apoptosis and suppressing proliferation, and act as a novel prognostic biomarker for bladder cancer.

\section{Introduction}

Bladder cancer is the fourth most common cancer in men and the ninth most common cancer in women worldwide (1). The majority of bladder cancers are urothelial cell carcinoma, which originate from the epithelial lining of the bladder wall (2). More than half of patients with bladder cancer are diagnosed with advanced stage cancer and have very poor prognosis (3). Despite improvement in treatment of bladder cancer, the incidence of the disease is still increasing (4). Although environmental and genetic factors have been demonstrated to have important roles in the development of bladder cancer, the molecular mechanisms involved in the initiation and progression of the disease remain unclear. Investigating the underlying mechanisms of bladder cancer is instrumental for providing better treatment and development of novel therapeutic agents.

The tumor necrosis factor receptor superfamily member 14 (TNFRSF14) gene, also known as herpes virus entry mediator, is located on the short arm of chromosome 1p36 and encodes a type I trans membrane molecule that serves as a molecular switch by interacting with different ligands to regulate a series of immune responses (5). Activation of TNFRSF14 is involved in the development of various tumor types, and may be used for assessing prognosis as it is closely associated with cancer growth and metastasis $(6,7)$. In a previous study, reduced TNFRSF14 expression was suggested to contribute to the pathobiology of classical Hodgkin lymphoma (8). Another previous study indicated that TNFRSF14 deficiency promotes development of follicular lymphoma in vivo and establishes a favorable immune environment (9). Cheung et al (10) reported that somatic TNFRSF14 mutations are associated with worse prognosis of follicular lymphoma. However, the expression levels of TNFRSF14 in other types of cancer types have been inconsistent. To the best of our knowledge, there is no published evidence on the expression of TNFRSF14 in bladder cancer tissues and its association with clinicopathological parameters remains poorly understood. 
In the present study, the aim was to investigate the expression levels of TNFRSF14 in bladder cancer and evaluate its clinical significance. In addition, the molecular mechanism of TNFRSF14 in regulating progression of bladder cancer was examined. The results indicated that increased expression of TNFRSF14 inhibited bladder cancer proliferation by promoting apoptosis.

\section{Materials and methods}

Database. The Cancer Genome Atlas (TCGA) database was utilized to obtain clinical information and Level 3 RNA sequencing (RNA-Seq) data. The search terms used in TCGA contained the following keywords: 'cases', 'primary site', 'bladder' and 'TCGA-BLCA'. Based on the above search terms, the clinical information and Level 3 RNA-Seq data were obtained from 404 bladder cancer samples and 28 normal bladder tissue samples (11). TNFRSF14 expression levels in normal and bladder cancer tissue were analyzed using the limma package (version 3.32.8) from Bioconductor (12).

Cell cultures. T24 and SW780 bladder cancer cell lines, and SV-HUC-1 normal human bladder epithelium cell line were purchased from the Shanghai Cell Bank at the Chinese Academy of Sciences (Shanghai, China). SV-HUC-1 cells were cultured in Corning ${ }^{\mathrm{TM}}$ cellgro $^{\mathrm{TM}} \mathrm{F} 12 \mathrm{~K}$ (Thermo Fisher Scientific, Inc., Waltham, MA, USA) supplemented with $10 \%$ fetal bovine serum (FBS; Gibco; Thermo Fisher Scientific, Inc.). T24 and SW780 bladder cancer cell lines, and SV-HUC-1 cells were cultured in a humidified atmosphere of $5 \% \mathrm{CO}_{2}$ at $37^{\circ} \mathrm{C}$. The EJ-M3 cell line was established from the EJ cell line, which has been demonstrated to be a T24 derivative/contaminants. EJ-M3 cells are essentially highly invasive T24 derivative cells (13) and were obtained from EJ cells (14). EJ-M3 cells were generated, according to the methods described by Girnita et al (15). Briefly, 24-well plates were filled with media containing chemokine, and Transwell inserts coated with $100 \mu \mathrm{g}$ Matrigel were placed into the wells and $3 \times 10^{5}$ EJ cells were added onto the membrane. After $7 \mathrm{~h}$ in culture, the insert was removed and cells invading the lower chamber were further cultured. The medium was changed after 4-6 days. After reaching lamellar fusion, the cells were digested and subcultured. This process was repeated three times and each time the amount of Matrigel coating on the insert membrane was increased by $50 \mu \mathrm{g}$. Cells that were retained following this selection process were identified highly invasive EJ-M3 cells. These cancer cells were cultured in RPMI-1640 (HyClone; GE Healthcare, Chicago, IL, USA) supplemented with 10\% FBS (Gibco; Thermo Fisher Scientific, Inc.), $100 \mathrm{U} / \mathrm{ml}$ penicillin, and $0.1 \mathrm{mg} / \mathrm{ml}$ streptomycin (Sigma-Aldrich; Merck KGaA, Darmstadt, Germany). Cells were cultured at $37^{\circ} \mathrm{C}$ in a humidified atmosphere containing $5 \% \mathrm{CO}_{2}$ until $70-80 \%$ confluence was reached. Non-adherent cells were washed away after 3 days of culture and adherent cells were fed with fresh complete medium. The cells were subcultured at a 1:2 split ratio after reaching confluence.

Transient transfection and small interfering RNA (siRNA) transfection. Cells were cultured in 6-well plates in medium without antibiotics for $24 \mathrm{~h}$ prior to transfection, resulting in
$70-80 \%$ confluence. For transient transfection, the TNFRSF14 gene was subcloned into a pSG5-HA vector, generating pSG5-HA-TNFRSF14. The empty vector was used as a negative control for transfection. Cells were transfected by Lipofectamine ${ }^{\circledR} 2000$ (Invitrogen; Thermo Fisher Scientific, Inc.) according to the manufacturer's protocol. Specifically, cells were cultured in fresh medium without antibiotics, and when the T2 4 cells reached 70-80\% confluence, $4 \mu \mathrm{g}$ DNA (pSG5-HA-TNFRSF14) was combined with 250 $\mu$ 1 Opti-MEM ${ }^{\circledR}$ medium (Invitrogen; Thermo Fisher Scientific, Inc.) and $10 \mu \mathrm{l}$ Lipofectamine $^{\circledR} 2000$ was diluted in $240 \mu$ l Opti-MEM ${ }^{\circledR}$, which were incubated at room temperature for $5 \mathrm{~min}$. DNA/Opti-MEM ${ }^{\circledR}$ and Lipofectamine ${ }^{\circledR}$ 2000/Opti-MEM ${ }^{\circledR}$ were combined and incubated at room temperature for $20 \mathrm{~min}$. A total of $500 \mu \mathrm{l}$ plasmid/Lipofectamine ${ }^{\circledR}$ complex was added to the cells, which were cultured at $37^{\circ} \mathrm{C}$. Following $24 \mathrm{~h}$, the transfection medium was replaced with fresh complete medium and cells were harvested for analysis of proliferation and other experiments.

siRNA against TNFRSF14 (si-TNFRSF14; cat. no. stQ0003897-1) and negative control (si-NC; cat. no. siN05815122147) were produced and purchased from Guangzhou RiboBio Co., Ltd. (Guangzhou, China). The negative control siRNA does not match any known mammalian GenBank sequences. Cells were seeded at a density of $3 \times 10^{5} /$ well in 6-well plates overnight and transfected with siRNA or si-NC at a final concentration of $100 \mathrm{nM}$ using Lipofectamine ${ }^{\circledR} 2000$ (Invitrogen; Thermo Fisher Scientific, Inc.). Following $24 \mathrm{~h}$ of transfection, reverse transcription-quantitative polymerase chain reaction (RT-qPCR) was used to determine transfection efficiency.

RNA extraction and RT-qPCR. RNA was extracted from cells using TRIzol ${ }^{\circledR}$ reagent (Invitrogen; Thermo Fisher Scientific, Inc.).cDNA was generated using PrimeScriptRT reagent kit (cat. no. RR037A; Takara Biotechnology Co., Ltd., Dalian, China) at $37^{\circ} \mathrm{C}$ for $15 \mathrm{~min}$. Synthesized first-strand cDNA was used as template, and GAPDH was applied for normalization. Primer sequences were used as follows: TNFRSF14 forward, 5'-CCA AGTGCAGTCCAGGTTAT-3' and reverse, 5'-ATTGAGGTG GGCAATGTAGG-3'; GAPDH forward, 5'-GGTGTGAAC CATGAGAAGTATGA-3' and reverse, 5'-GAGTCCTTCCAC GATACCAAAG-3'. The RT-qPCR reaction was performed at $95^{\circ} \mathrm{C}$ for $5 \mathrm{~min}$, followed by 40 cycles of $95^{\circ} \mathrm{C}$ for $30 \mathrm{sec}, 60^{\circ} \mathrm{C}$ for $45 \mathrm{sec}$ and $72^{\circ} \mathrm{C}$ for $30 \mathrm{~min}$ using an ABI 7500 real-time PCR system (Applied Biosystems; Thermo Fisher Scientific, Inc.) using SYBR Select Master Mix (Applied Biosystems; Thermo Fisher Scientific, Inc.) following the manufacturer's protocol The relative expression levels of TNFRSF14 were analyzed and normalized to GAPDH using the $2^{-\Delta \Delta \mathrm{Cq}}$ method (16).

Cell proliferation analysis. The Cell Counting kit-8 (CCK-8) assay is a sensitive and accurate method for determining cell viability. In the present study, cell proliferation was evaluated using the CCK-8 assay at 24, 48 and $72 \mathrm{~h}$ after transfection, according to a previous study (17). Briefly, cells in the TNFRSF14 or si-TNFRSF14 transfection group and control transfection group were cultured in $100 \mu 1$ RPMI-1640 supplemented with $10 \%$ FBS in 96 -well plates and incubated at $37^{\circ} \mathrm{C}$ 
for 3 days. Subsequently, CCK-8 dye (BestBio, Shanghai, China), diluted 1:10 in cell culture medium, was added to each well and incubated for $2 \mathrm{~h}$ at $37^{\circ} \mathrm{C}$. The absorbance was read using a microplate reader set at $450 \mathrm{~nm}$. The $450 \mathrm{~nm}$ optical density value is proportional to the total number of live cells and was used to assess cell viability in transfected vs. control transfected cells. Triplicate wells were used and experiments were repeated at least three times.

Western blot analysis. Total protein was extracted from transfected and control transfected cells using $1 \mathrm{mM}$ phenylmethylsulfonyl fluoride in $1 \mathrm{ml}$ ice-cold radioimmunoprecipitation assay buffer (Beyotime Institute of Biotechnology, Haimen, China). Subsequently, protein centration was measured using the bicinchoninic acid assay. Denatured proteins $(20 \mu \mathrm{g})$ were separated using $12 \%$ SDS-PAGE and transferred onto Immobilon-P polyvinylidene fluoride membranes (EMD Millipore, Billerica, MA, USA). Membranes were blocked for $2 \mathrm{~h}$ with $5 \%$ skimmed milk powder in Tris-buffered saline containing $0.1 \%$ Tween-20 (TBST) and incubated overnight at $4^{\circ} \mathrm{C}$ with the following primary antibodies: Anti-protein kinase B (AKT; 1:1,000; cat. no. SAB4500797), anti-phosphorylated (p)-AKT (1:1,000; cat. no. SAB4301414), anti-P70 S6 kinase (P70; 1:1,000; cat. no. SAB4502683), anti-active caspase3-p17 (1:1,000; cat. no. SAB4503294) and anti-GAPDH (1:5,000; cat. no. SAB2108266; Sigma-Aldrich; Merck KGaA). Subsequently, membranes were washed three times with TBST at room temperature, followed by incubation with an horseradish peroxidase-conjugated anti-rabbit IgG secondary antibody (1:5,000; cat. no. sc-2004; Santa Cruz Biotechnology, Inc., Dallas, TX, USA) at room temperature for $2 \mathrm{~h}$. Membranes were washed a final time with TBST. GAPDH was used as the loading control. All bands were detected using Enhanced Chemiluminescent Western Blotting Detection kit (GE Healthcare Life Sciences, Little Chalfont, UK). Imaging and quantification of protein bands was conducted using the Bio-Rad Quantity One 1-D Analysis software version 4.6.9 (Bio-Rad Laboratories, Inc., Hercules, CA, USA).

Apoptosis assay. Following $24 \mathrm{~h}$ of transfection, cells were harvested by trypsinization, washed with pre-cooled PBS and fixed overnight with $70 \%$ ethanol at $-20^{\circ} \mathrm{C}$. T24 or EJ-M3 cells were washed twice, resuspended in PBS containing $0.1 \mathrm{mg} / \mathrm{ml} \mathrm{RNase} \mathrm{A}$ and $0.1 \%$ Triton X-100 in the dark for $30 \mathrm{~min}$ at $37^{\circ} \mathrm{C}$. A total of $1 \times 10^{6}$ cells were double stained with Annexin $\mathrm{V}$-fluorescein isothiocyanate (FITC) and propidium iodide (PI) using the Apoptosis Detection kit (BD Biosciences, Franklin Lakes, NJ, USA), according to the manufacturer's protocol. The stained cells were acquired on a FACScan flow cytometer (BD Biosciences) equipped with a $488 \mathrm{~nm}$ argon laser and the data were analyzed using Cell Quest software version 6.0 (BD Biosciences). Cells were categorized into viable (Annexin $\mathrm{V}-\mathrm{FITC}^{-} / \mathrm{PI}^{-}$), dead (Annexin $\mathrm{V}-\mathrm{FITC}^{-} / \mathrm{PI}^{+}$), early apoptotic (Annexin $\mathrm{V}-\mathrm{FITC}^{+} / \mathrm{PI}^{-}$) or apoptotic cells (Annexin $\mathrm{V}-\mathrm{FITC}^{+} / \mathrm{PI}^{+}$). The percentage of apoptotic cells in the experiment group was compared with the control transfection group. All the samples were measured in triplicate.

Statistical analysis. In the present study, SPSS software version 18.0 (SPSS, Inc., Chicago, IL, USA) was used to conduct all statistical analyses. Each assay was performed at least three times. The data are presented as the mean \pm standard deviation. The means between two groups were compared using Student's t-test. One-way analysis of variance followed by Student-Newman-Keuls post hoc test was used to compare the means of multiple groups. The Kaplan-Meier method was used to evaluate the prognostic value of TNFRSF14 in bladder cancer, and the Mantel-Cox log-rank test was used to determine the statistical significance of difference between survival curves. $\mathrm{P}<0.05$ was considered to indicate a statistically significant difference.

\section{Results}

Downregulation of TNFRSF14 expression levels in human bladder cancer tissues is associated with poor prognosis. The association between TNFRSF14 expression levels and prognosis of bladder cancer was assessed using RNA-Seq data from the TCGA database. The results indicated that TNFRSF14 expression levels were downregulated in the bladder tissues of patients with bladder cancer compared with healthy controls (Fig. 1A; P<0.05). Based on the median value of TNFRSF14 expression, patients were divided into TNFRSF14 low and TNFRSF14 high expression groups. As shown in Fig. 1B, the overall survival was significantly longer in the TNFRSF14 high expression group compared with in the TNFRSF14 low expression group.

TNFRSF14 expression levels are downregulated in bladder cancer cell lines. TNFRSF14 expression levels in three human bladder cancer cell lines (T24, SW780 and EJ-M3) and a normal human bladder epithelium cell line (SV-HUC-1) were measured by RT-qPCR. The results demonstrated TNFRSF14 expression levels were lower in all bladder cancer cell lines compared with in the SV-HUC-1 cell line (Fig. 2). Notably, the expression levels of TNFRSF14 in T24 cells were lower compared with in SW780 and EJ-M3 cells. Therefore, T24 cells were used to perform overexpression experiments, whereas EJ-M3 cells were used to conduct silencing assays.

CCK-8 analysis of cell viability. To investigate the role of TNFRSF14 in T24 or EJ-M3 cell proliferation, overexpression and silencing experiments were conducted using pSG5-HA-TNFRSF14 and si-TNFRSF14, respectively. RT-qPCR analysis was conducted $72 \mathrm{~h}$ after transfection to measure transfection efficacy. The results in Fig. 3A indicated that TNFRSF14 expression levels were significantly increased in $\mathrm{T} 24$ cells after transfection $(\mathrm{P}<0.05)$. As demonstrated in Fig. 4A, TNFRSF14 expression levels were decreased in EJ-M3 cells after gene silencing.

Subsequently, the effect of TNFRSF14 overexpression on cell proliferation was evaluated using the CCK-8 assay at 24, 48 and $72 \mathrm{~h}$ after transfection. As demonstrated in Fig. 3B, TNFRSF14 transfection led to a reduction in cell viability of T24 cells, which was statistically significant compared with control transfection group after $72 \mathrm{~h}$ of transfection ( $\mathrm{P}<0.05$; Fig. 3B). The results indicated TNFRSF14 overexpression decreased cell proliferation of bladder cancer cells.

In addition, CCK-8 assay was used to measure the effect of TNFRSF14 silencing on cell proliferation of EJ-M3 


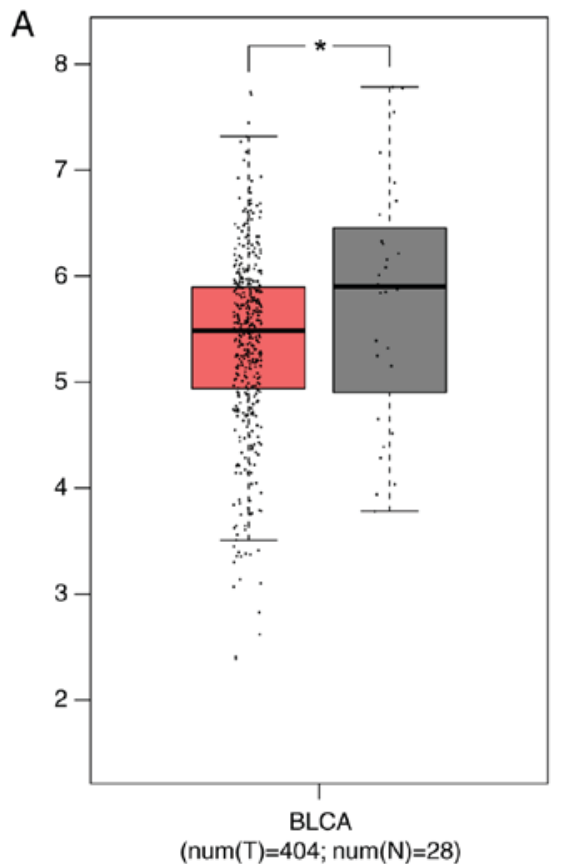

B

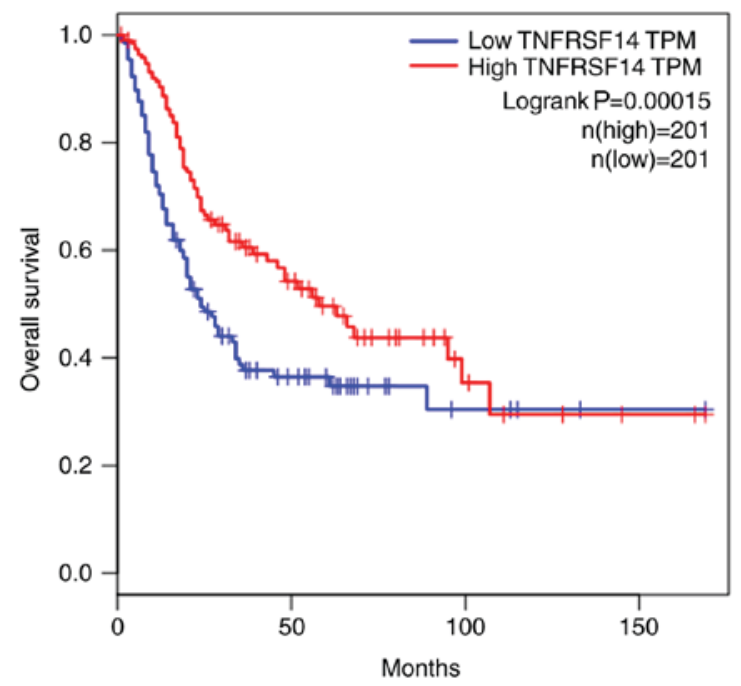

Figure 1. TNFRSF14 is downregulated in bladder cancer and serves as an indicator for prognosis. (A) Expression levels of TNFRSF14 in bladder cancer tissue (red; n=404) and normal control tissue (gray; $\mathrm{n}=28$ ), using The Cancer Genome Atlas data. (B) Kaplan-Meier survival analysis for patients with bladder cancer exhibiting low or high TNFRSF14 expression. In survival analysis, patients were divided into high and low expression groups based on the median value of TNFRSF14 expression levels. "P<0.05. BLCA, bladder cancer; $\mathrm{T}$, tumor; $\mathrm{N}$, normal tissue; TNFRSF14, tumor necrosis factor receptor superfamily member 14; TPM, transcripts per million.

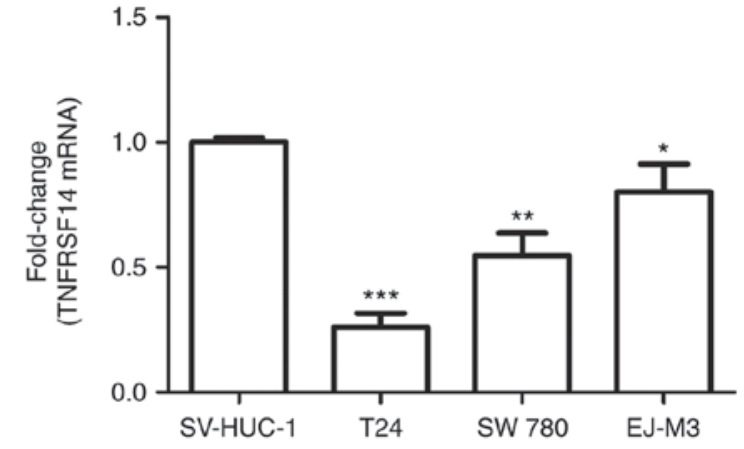

Figure 2. Reverse transcription-quantitative polymerase chain reaction analysis of TNFRSF14 expression levels in three human bladder cancer cell lines (T24, SW780 and EJ-M3) and a normal human SV-HUC-1 bladder epithelium cell line. TNFRSF14 expression levels were normalized against GAPDH. ${ }^{*} \mathrm{P}<0.05,{ }^{* *} \mathrm{P}<0.01,{ }^{* * *} \mathrm{P}<0.001$ vs. SV-HUC-1. TNFRSF14, tumor necrosis factor receptor superfamily member 14 .

cells after 24, 48 and 72 h. Compared with control transfected cells, TNFRSF14 silencing resulted in increased cell viability of EJ-M3 cells, particularly at $72 \mathrm{~h}$ after transfection $(\mathrm{P}<0.05$; Fig. 4B). The results indicated that TNFRSF14 silencing increased cell proliferation of bladder cancer cells.

Apoptosis assay. To evaluate the effect of alterations in TNFRSF14 expression levels on apoptosis of T24 or EJ-M3 cells, Annexin V-FITC and PI double staining was utilized to detect apoptotic cells, including early (Annexin $\mathrm{V}^{-F_{T C}} / \mathrm{PI}^{-}$) and late apoptotic (Annexin V-FITC ${ }^{+} / \mathrm{PI}^{+}$) cells. As presented in Fig. 5, TNFRSF14 overexpression promoted increased apoptosis in transfected cells compared with in control cells (5.52 vs. $1 ; \mathrm{P}<0.05$ ), indicating that TNFRSF14 overexpression may have a direct therapeutic effect against the human T24 bladder cancer cell line. As demonstrated in Fig. 6, TNFRSF14 silencing decreased apoptosis in transfected cells compared with in control cells $(0.51$ vs. $1 ; \mathrm{P}<0.05)$. The results suggested that suppression of cell viability following TNFRSF14 overexpression was associated with induction of cell apoptosis.

Phosphatidylinositol 3-kinase (PI3K) signaling pathway. To determine whether abnormalities in TNFRSF14 expression levels affect PI3K signaling, numerous proteins were detected using western blot analysis, including p-AKT, caspase3-p17 and P70. As demonstrated in Fig. 7, TNFRSF14 overexpression significantly increased caspase3-p17 expression levels in T24 cells $(\mathrm{P}<0.05)$, which was in line with the cell apoptosis results obtained in the present study. Furthermore, TNFRSF14 overexpression significantly decreased p-AKT and P70 expression levels in the T24 bladder cancer cell line (Fig. 7B, $\mathrm{P}<0.05)$. Conversely, TNFRSF14 silencing significantly decreased caspase3-p17 expression levels in EJ-M3 cells $(\mathrm{P}<0.05)$, which was again in line with the cell apoptosis results. Furthermore, TNFRSF14 silencing significantly increased p-AKT $(\mathrm{P}<0.01)$ and $\mathrm{P} 70(\mathrm{P}<0.05)$ expression levels in the EJ-M3 bladder cancer cell line (Fig. 8).

\section{Discussion}

Human bladder cancer is one of the most lethal human cancers worldwide. Systematic chemotherapy and surgery are the primary treatment options for bladder cancer $(18,19)$; however, bladder cancer cells frequently develop drug resistance and $\sim 50 \%$ of patients with advanced bladder cancer do not respond to chemotherapy. Chemopreventative drugs may 

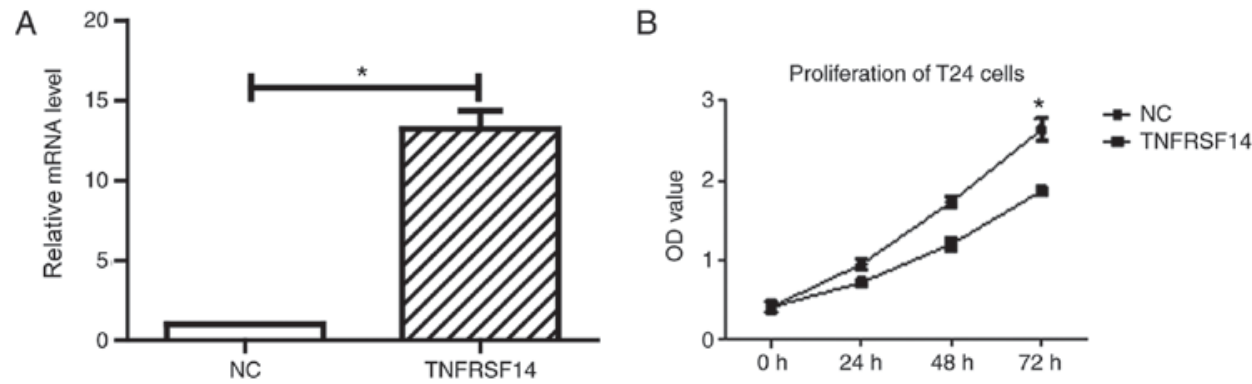

Figure 3. TNFRSF14 overexpression inhibits cell viability. (A) T24 cells were transfected with pSG5-HA-TNFRSF14 and TNFRSF14 expression levels were evaluated using reverse transcription-quantitative polymerase chain reaction. (B) Cell proliferation was measured by Cell Counting kit-8 assay. Each assay was performed at least three times. The data are presented as the mean \pm standard deviation. "P<0.05 vs. NC. NC, negative control; OD, optical density; TNFRSF14, tumor necrosis factor receptor superfamily member 14 .
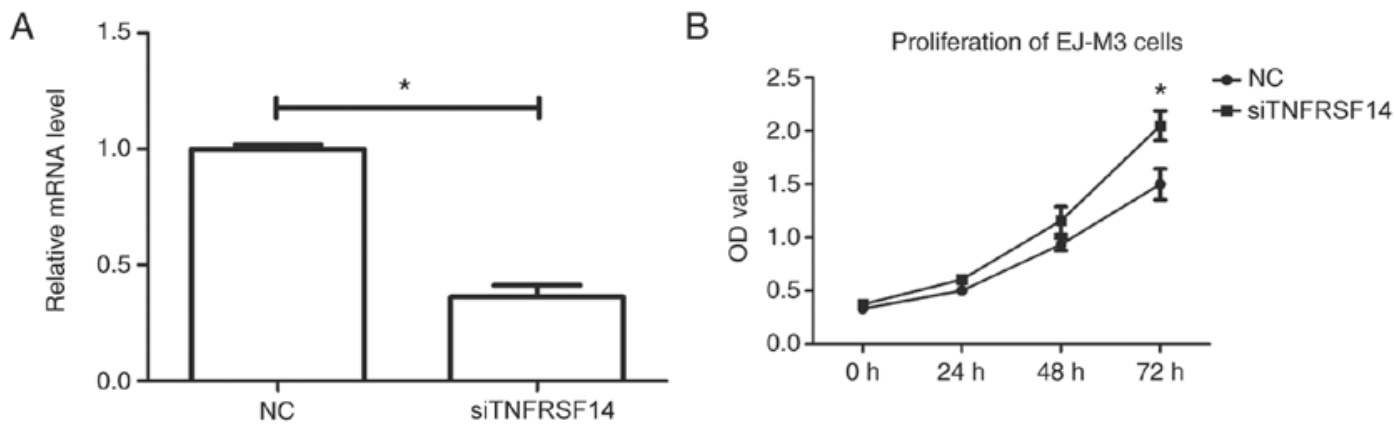

Figure 4. TNFRSF14 silencing enhances cell viability. (A) EJ-M3 cells were transfected with si-TNFRSF14 and TNFRSF14 expression levels were evaluated using reverse transcription-quantitative polymerase chain reaction. (B) Cell proliferation ability was determined using Cell Counting kit- 8 assay. ${ }^{*} \mathrm{P}<0.05$ vs. NC. NC, negative control; si, small interfering RNA; OD, optical density; TNFRSF14, tumor necrosis factor receptor superfamily member 14 .

A
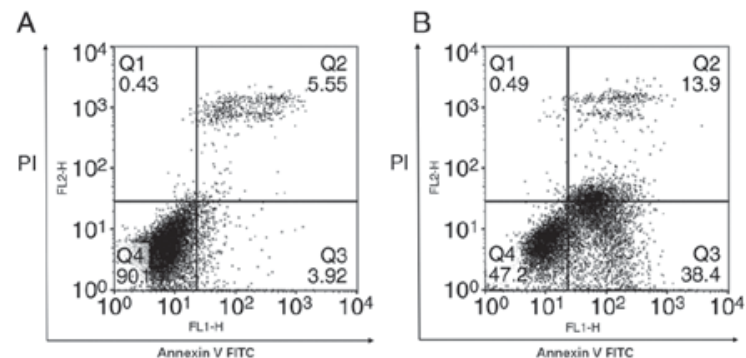

C

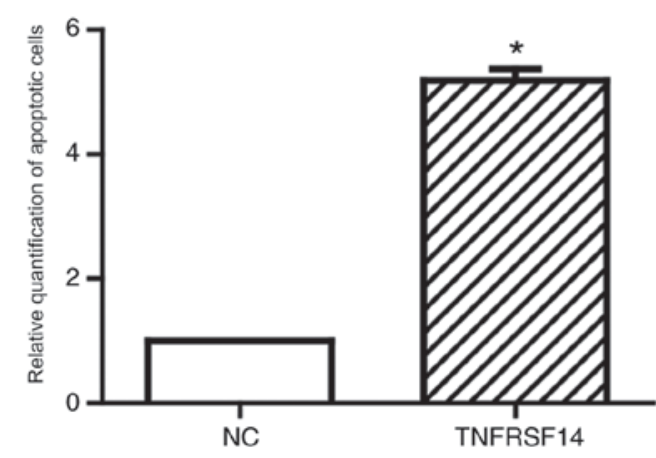

Figure 5. TNFRSF14 overexpression in T24 cells increases apoptosis. Flow cytometry dot plots of (A) control and (B) pSG5-HA-TNFRSF14-transfected T24 cells stained with Annexin V-FITC and propidium iodide, $72 \mathrm{~h}$ after transfection. The proportion of the cell number is presented in each quadrant. In each plot, Q1 denotes dead cells, Q2 represents late apoptotic cells, Q3 denotes early apoptotic cells and Q4 represents viable cells. (C) Relative quantification of apoptotic cells. Quantification of apoptotic cells in NC group set as 1. All experiments were performed in triplicate with three technical replicates. The data are presented as the means \pm standard deviation. ${ }^{*} \mathrm{P}<0.05$ vs. NC. NC, negative control; TNFRSF14, tumor necrosis factor receptor superfamily member 14 .
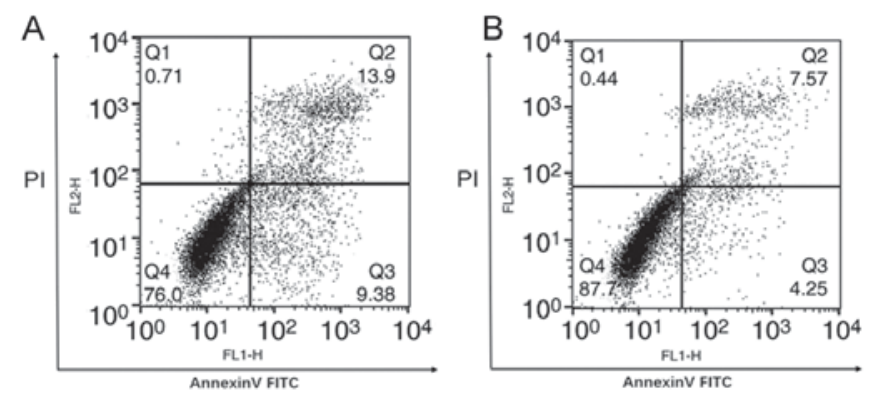

C

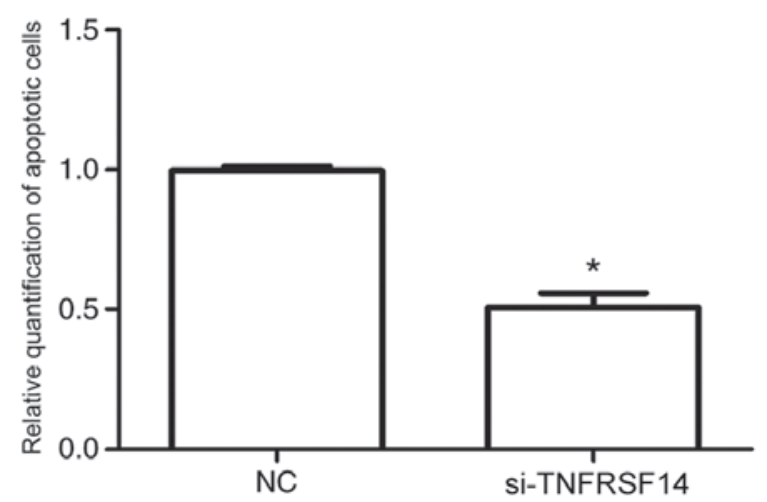

Figure 6.TNFRSF14 silencing in EJ-M3 decreases apoptosis. Flow cytometry dot plots of (A) control and (B) transfected EJ-M3 cells stained with Annexin V-FITC and PI, $72 \mathrm{~h}$ after transfection. (C) Relative quantification of apoptotic cells. Quantification of apoptotic cells in NC group set as 1. All experiments were performed in triplicate with three technical replicates. The data are presented as the means \pm standard deviation. ${ }^{*} \mathrm{P}<0.05$ vs. NC. $\mathrm{NC}$, negative control; si, small interfering RNA; TNFRSF14, tumor necrosis factor receptor superfamily member 14 . 

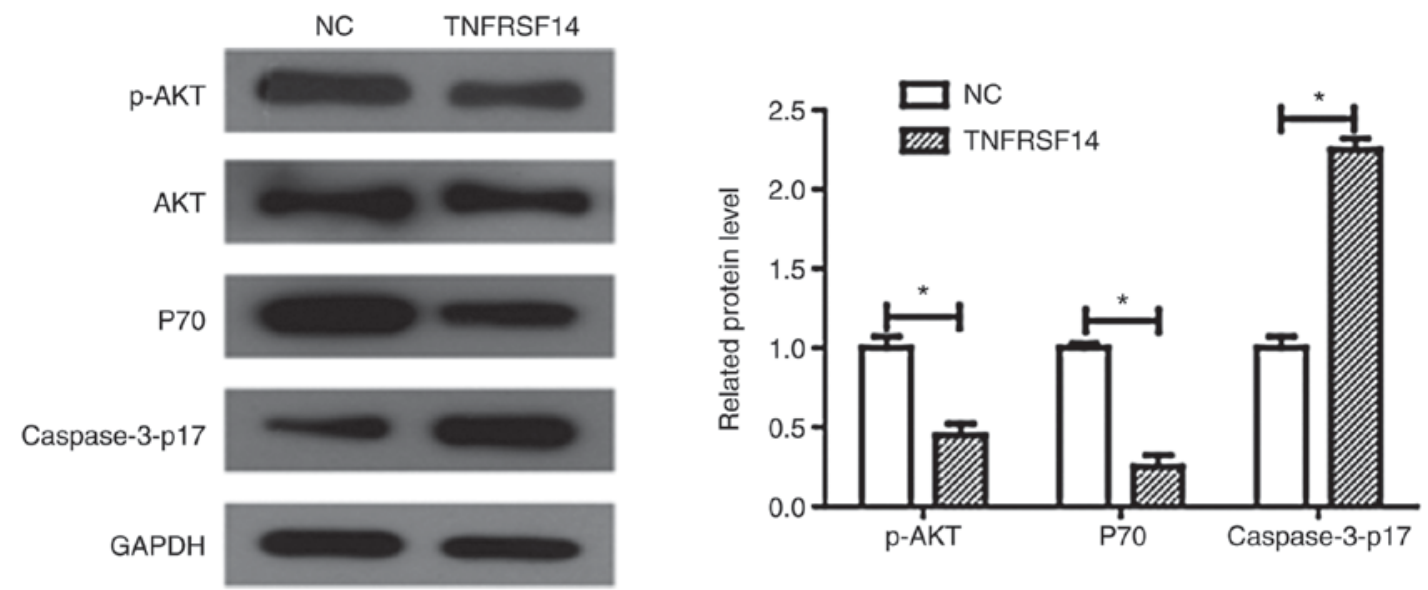

Figure 7. Western blot analysis of AKT, p-AKT, P70 and caspase3-p17 in T24 cells $72 \mathrm{~h}$ after transfection with TNFRSF14 vector, and quantification of band intensity. Protein expression levels were normalized against GAPDH. The data are presented as the means \pm standard deviation. ${ }^{*} \mathrm{P}<0.05$ vs. NC. AKT, protein kinase B; NC, negative control; p-, phosphorylated-; P70, P70 S6 kinase; TNFRSF14, tumor necrosis factor receptor superfamily member 14.
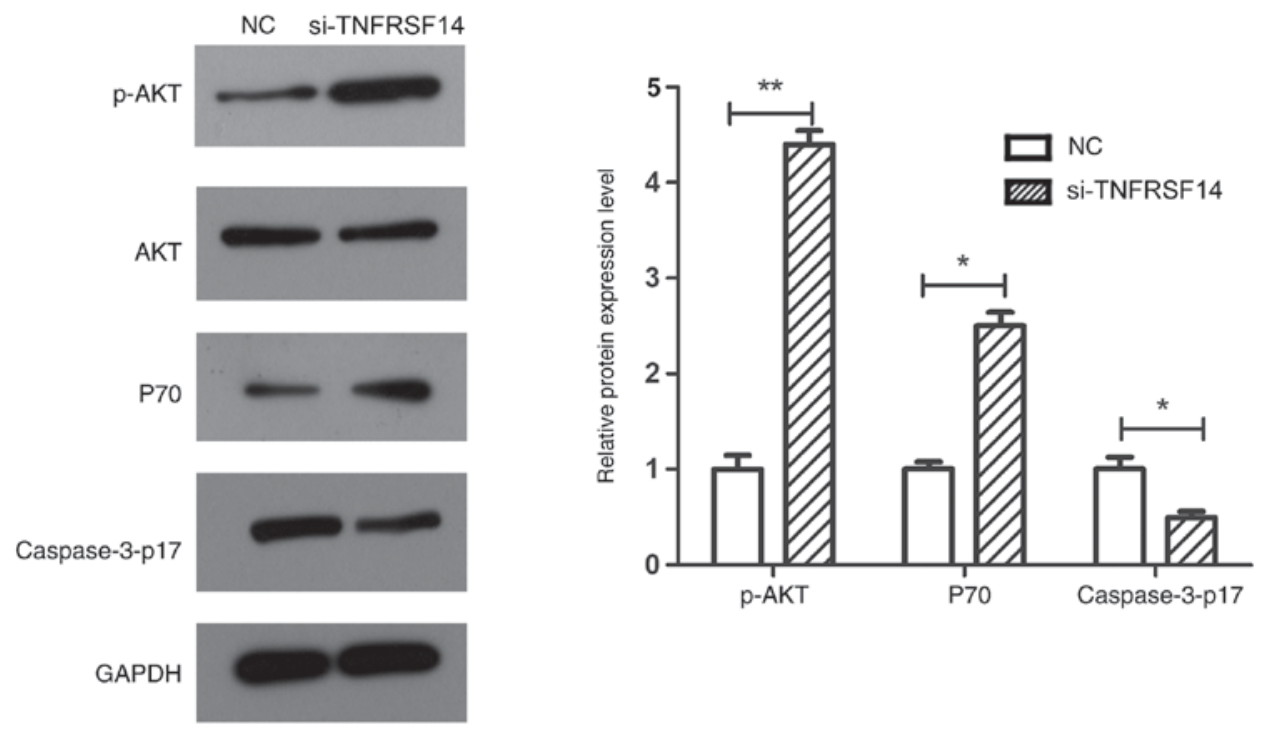

Figure 8. Western blot analysis of AKT, p-AKT, P70 and caspase3-p17 in EJ-M3 cells $72 \mathrm{~h}$ after transfection with si-TNFRSF14, and quantification of band intensity. The data are presented as the means \pm standard deviation. Protein expression levels were normalized against GAPDH. ${ }^{*} \mathrm{P}<0.05,{ }^{* * *} \mathrm{P}<0.01$ vs. NC. AKT, protein kinase B; NC, negative control; p-, phosphorylated-; P70, P70 S6 kinase; TNFRSF14, tumor necrosis factor receptor superfamily member 14.

inhibit and delay the initiation of cancer types via various mechanisms, including anti-proliferation or pro-apoptosis. Notably, TNFRSF14 activation has been demonstrated to inhibit proliferation of adenocarcinoma cells (20) and regulate apoptosis (21), suggesting a potential tumor suppressive role. To the best of our knowledge, the role of TNFRSF14 in bladder cancer has not yet been examined, and investigating its underlying mechanisms of action may aid in the development of novel diagnostic and therapeutic approaches to bladder cancer.

In the present study, it was demonstrated that expression levels of TNFRSF14 were decreased in bladder cancer tissue compared with negative control tissue, using the TCGA database. Patients with bladder cancer exhibiting low expression levels of TNFRSF14 had poorer prognosis compared with those exhibiting high expression levels of TNFRSF14. Similarly, TNFRSF14 expression levels were additionally reduced in bladder cancer cell lines. Overexpression of TNFRSF14 in T24 cells stimulated apoptosis and inhibited proliferation in vitro, which may indicate the potential protective effects of TNFRSF14 in bladder cancer. Conversely, TNFRSF14 knockdown in EJ-M3 cells enhanced cell proliferation, inhibited cell apoptosis, increased the expression levels of p-AKT and P70, and decreased the expression levels of caspase3-p17. Overall, the results demonstrated that TNFRSF14 may act as a tumor suppressor gene in bladder cancer, which is in line with a previous study (20).

Apoptosis is a form of programmed cell death, which serves a key role in tissue homeostasis and development in multicellular organisms (22). An imbalance between cell proliferation and apoptosis may lead to fatal diseases, including cancer (23). In various cell-based models of cancer, cell death triggered by a stimulus may be switched off by suppressing control points in the cell death pathway; for example, inhibition of caspase activation (24). Activation of caspase-3 results in cleavage of numerous proteins, including poly(ADP-ribose) polymerase (PARP). PARP is a nuclear DNA-binding zinc finger protein, 
which is important for DNA repair as well as other cellular processes, including cell differentiation, proliferation and apoptosis (25). Cleaved PARP is a primary indicator of apoptosis. In the present study, the results demonstrated that TNFRSF14 overexpression in T24 cells increased the expression levels of caspase- 3 and corresponded to an increased number of apoptotic cells. Therefore, TNFRSF14 may block bladder cancer progression by increasing the expression levels of caspase-3, thereby promoting apoptosis and suppressing proliferation of bladder cancer cells.

The PI3K pathway has a pivotal role in cell growth, proliferation and survival (26), and is upregulated in a number of different types of cancer (27). Notably, the PI3K pathway is activated in bladder cancer (28). AKT occupies an important regulatory node in the PI3K pathway, below which the pathway branches significantly to influence a wide range of cellular processes that promote cell-cycle progression, cell growth and resistance to apoptosis. PI3K-AKT signaling has been demonstrated to have an important role in transforming growth factor $\beta$-mediated epithelial-to-mesenchymal transition (29), which is a key process in bladder cancer development (30). A previous study suggested that downregulation of $\mathrm{p}-\mathrm{AKT}$ induces caspase-3-dependent apoptosis in cancer cells $(31,32)$, which is consistent with the results obtained in the present study. TNFRSF14 overexpression significantly increased the expression levels of caspase-3, but significantly decreased the expression levels of p-AKT and P70 in T24 bladder cancer cells. Silencing of TNFRSF14 yielded opposite results. The results suggested that TNFRSF14-mediated apoptosis is regulated by a caspase-dependent cascade that blocks PI3K/AKT signaling and activates other specific signaling pathways.

In conclusion, to the best of our knowledge, this is the first study of its kind to investigate the prognostic value of TNFRSF14 in bladder cancer. The results indicated TNFRSF14 expression levels were decreased in bladder cancer tissue compared with in normal control tissues using the TCGA database. Patients with bladder cancer exhibiting low expression levels of TNFRSF14 had worse prognosis compared with those exhibiting high expression levels of TNFRSF14. Therefore, TNFRSF14 may act as a tumor suppressor in bladder cancer and serve as a novel prognostic biomarker. Nevertheless, there are limitations to the present study. The association between TNFRSF14 expression levels and prognosis of bladder cancer was determined using RNA-Seq data from the TCGA database. Further studies using patient tissues are required to validate the present findings.

\section{Acknowledgements}

Not applicable.

\section{Funding}

No funding was received.

\section{Availability of data and materials}

The datasets used and/or analyzed during the current study are available from the corresponding author on reasonable request.

\section{Authors' contributions}

YZ performed the experiments, analyzed the data and drafted the manuscript; M-YL conceived the study and revised the manuscript. All authors read and approved the final manuscript.

\section{Ethics approval and consent to participate}

Not applicable.

\section{Patient consent for publication}

Not applicable.

\section{Competing interests}

The authors declare that they have no competing interests.

\section{References}

1. Luo M, Li Z, Wang W, Zeng Y, Liu Z and Qiu J: Long non-coding RNA H19 increases bladder cancer metastasis by associating with EZH2 and inhibiting E-cadherin expression. Cancer Lett 333: 213-221, 2013.

2. Bo J, Yang G, Huo K, Jiang H, Zhang L, Liu D and Huang Y: microRNA-203 suppresses bladder cancer development by repressing bcl-w expression. FEBS J 278: 786-292, 2011.

3. Stein JP, Grossfeld GD, Ginsberg DA, Esrig D, Freeman JA, Figueroa AJ, Skinner DG and Cote RJ: Prognostic markers in bladder cancer: A contemporary review of the literature. J Urol 160: 645-659, 1998.

4. Laufer M, Ramalingam S, Schoenberg MP, Haisfieldwolf ME, Zuhowski EG, Trueheart IN, Eisenberger MA, Nativ O and Egorin MJ: Intravesical gemcitabine therapy for superficial transitional cell carcinoma of the bladder: A phase I and pharmacokinetic study. J Clin Oncol 21: 697-703, 2003.

5. Shui JW, Steinberg MW and Kronenberg M: Regulation of inflammation, autoimmunity and infection immunity by HVEM-BTLA signaling. J Leukoc Biol 89: 517-523, 2011.

6. Bolyard C, Ji YY, Wang PY, Saini U, Rath KS, Cripe T, Zhang J, Selvendiran K and Kaur B: Doxorubicin synergizes with 34.5 ENVE to enhance antitumor efficacy against metastatic ovarian cancer. Clin Cancer Res 20: 6479-6494, 2014.

7. Shui JW, Larange A, Kim G, Vela JL, Zahner S, Cheroutre H and Kronenberg M: HVEM signalling at mucosal barriers provides host defence against pathogenic bacteria. Nature 488: 222-225, 2012.

8. Salipante SJ, Adey A, Thomas A, Lee C, Liu YJ, Kumar A, Lewis AP, Wu D, Fromm JR and Shendure J: Recurrent somatic loss of TNFRSF14 in classical Hodgkin lymphoma. Genes Chromosomes Cancer 55: 278-287, 2016.

9. Kotsiou E, Okosun J, Besley C, Iqbal S, Matthews J, Fitzgibbon J, Gribben JG and Davies JK: TNFRSF14 aberrations in follicular lymphoma increase clinically significant allogeneic T-cell responses. Blood 128: 72-81, 2016.

10. Cheung KJ, Johnson NA, Affleck JG, Severson T, Steidl C, Ben-Neriah S, Schein J, Morin RD, Moore R, Shah SP, et al: Acquired TNFRSF14 mutations in follicular lymphoma are associated with worse prognosis. Cancer Res 70: 9166-9174, 2010.

11. Cancer Genome Atlas Research Network: Comprehensive molecular characterization of urothelial bladder carcinoma. Nature 507: 315-322, 2014.

12. Ritchie ME, Phipson B, Wu D, Hu Y, Law CW, Shi W and Smyth GK: Limma powers differential expression analyses for RNA-sequencing and microarray studies. Nucleic Acids Res 43: e47, 2015 .

13. Capesdavis A, Theodosopoulos G, Atkin I, Drexler HG, Kohara A, MacLeod RA, Masters JR, Nakamura Y, Reid YA, Reddel RR and Freshney RI: Check your cultures! A list of cross-contaminated or misidentified cell lines. Int J Cancer 127: $1-8,2010$ 
14. Yang D, Wang $\mathrm{H}$, Wang J, Zhang $\mathrm{C}$ and $\mathrm{Xu} \mathrm{H}$ : Establishment of a fluorescent implantation metastasis model of bladder cancer and real-time microscopic detection in nude mice. Asian Pac J Cancer Prev 12: 393-396, 2011.

15. Girnita A, All-Ericsson C,Economou MA, Astrom K, Axelson M, Seregard S, Larsson O and Girnita L: The insulin-like growth factor-I receptor inhibitor picropodophyllin causes tumor regression and attenuates mechanisms involved in invasion of uveal melanoma cells. Clin Cancer Res 12: 1383-1391, 2006.

16. Livak KJ and Schmittgen TD: Analysis of relative gene expression data using real-time quantitative PCR and the 2(-Delta Delta C(T)) method. Methods 25: 402-408, 2001.

17. Zhang X, Qi H, Wang S, Feng L, Ji Y, Tao L, Li S and Wei Y: Cellular responses of aniline oligomers: A preliminary study. Toxicol Res 1: 201-205, 2012

18. van Rhijn BW, Burger M, Lotan Y, Solsona E, Stief CG, Sylvester RJ, Witjes JA and Zlotta AR: Recurrence and progression of disease in non-muscle-invasive bladder cancer: From epidemiology to treatment strategy. Eur Urol 56: 430-442, 2009.

19. Geng W, Ng KTP, Sun CKW, Yau WL, Liu XB, Cheng Q Poon RT, Lo CM, Man K and Fan ST: The role of proline rich tyrosine kinase 2(Pyk2) on cisplatin resistance in hepatocellular carcinoma. PLoS One 6: e27362, 2011.

20. Harrop JA, Mcdonnell PC, Brigham-Burke M, Lyn SD, Minton J, Tan KB, Dede K, Spampanato J, Silverman C, Hensley P, et al: Herpesvirus entry mediator ligand (HVEM-L), a novel ligand for HVEM/TR2, stimulates proliferation of T cells and inhibits HT29 cell growth. J Biol Chem 273: 27548-27556, 1998.

21. Gaur U and Aggarwal BB: Regulation of proliferation, survival and apoptosis by members of the TNF superfamily. Biochem Pharmacol 66: 1403-1408, 2003.

22. Hengartner MO: Biochemistry of apoptosis. Nature 407: 770-776, 2016.

23. Hail N Jr, Carter BZ, Konopleva M and Andreeff M: Apoptosis effector mechanisms: A requiem performed in different keys. Apoptosis 11: 889-904, 2006.
24. Hartmann A, Troadec J, Hunot S, Kikly K, Faucheux B, Mouatt-Prigent A, Ruberg M, Agid Y and Hirsch E: Caspase-8 is an effector in apoptotic death of dopaminergic neurons in parkinson's disease, but pathway inhibition results in neuronal necrosis. J Neurosci 21: 2247-2255, 2001.

25. Isabelle M, Moreel X, Gagné JP, Rouleau M, Ethier C, Gagné P, Hendzel MJ and Poirier GG: Investigation of PARP-1, PARP-2 and PARG interactomes by affinity-purification mass spectrometry. Proteome Sci 8: 22, 2010.

26. Cantley LC: The phosphoinositide 3-kinase pathway. Science 296: 1655-1657, 2002.

27. Shaw RJ and Cantley LC: Ras, PI(3)K and mTOR signalling controls tumour cell growth. Nature 441: 424-430, 2006.

28. Knowles MA, Platt FM, Ross RL and Hurst CD: Phosphatidylinositol 3-kinase (PI3K) pathway activation in bladder cancer. Cancer Metastasis Rev 28: 305-316, 2009.

29. Bakin AV, Tomlinson AK, Bhowmick NA, Moses HL and Arteaga CL: Phosphatidylinositol 3-kinase function is required for transforming growth factor $\beta$-mediated epithelial to mesenchymal transition and cell migration. J Biol Chem 275: 36803 , 2000.

30. Mcconkey DJ, Choi W, Marquis L, Martin F, Williams MB, Shah J, Svatek R, Das A, Adam L, Kamat A, et al: Role of epithelial-to-mesenchymal transition (EMT) in drug sensitivity and metastasis in bladder cancer. Cancer Metastasis Rev 28: 335-344, 2009.

31. Wang YB, Qin J, Zheng XY, Bai Y, Yang K and Xie LP: Diallyl trisulfide induces Bcl-2 and caspase-3-dependent apoptosis via downregulation of Akt phosphorylation in human T24 bladder cancer cells. Phytomedicine 17: 363-368, 2010.

32. Tang C, Lu YH, Xie JH, Wang F, Zou JN, Yang JS, Xing YY and Xi T: Downregulation of survivin and activation of caspase-3 through the PI3K/Akt pathway in ursolic acid-induced HepG2 cell apoptosis. Anticancer Drugs 20: 249-258, 2009. 